\title{
Detection of left atrial thrombi in man using indium-111 labelled autologous platelets
}

\author{
MAKOTO YAMADA, NORITAKE HOKI, KIYOMU ISHIKAWA, HIDEO YOSHIMA, \\ SEKIKEN HATA, NOBUKAZU OHKUBO, RYOUSUKE MATSUWAKA, KEIICHI \\ FURUBAYASHI, MITSUYA FUKUSHIMA, KENJI ONISHI, YOSHIO KOBAYASHI \\ From the Heart Centre, Osaka Prefectural Hospital, Osaka, Fapan
}

SUMMARY A scintigraphic technique using indium-111 labelled platelets to detect left atrial thrombi was used in 28 patients, 14 of whom had mitral valve disease and 14 combined valve disease. Imaging was performed in the anterior, right anterior oblique $\left(45^{\circ}\right)$, and left lateral views on the day of injection and thereafter at one or two day intervals for a maximum of four days. When scintiphotos obtained in two or three views 72 or 96 hours after the platelet injection showed "hot spot areas" within the left atrial pool and indium-111 activity in these areas did not decrease with time they were interpreted as positive for thrombi. Of 28 patients, seven had positive platelet images by this criterion; of these, three underwent surgery and were found to have left atrial thrombi. One patient died, and a thrombus was found at necropsy. The remaining 21 patients had normal scintiphotos; of these, seven had no thrombi at operation and one had false negative images.

The diagnostic accuracy of platelet scintigraphy by this criterion of positivity in the 12 patients in whom surgical or postmortem confirmation of thrombi could be obtained was $92 \%$. These results indicate that this technique is a promising method for detecting active left atrial thrombi.

Left atrial thrombi occur in patients with mitral valve disease and may lead to catastrophic embolic events. In studies of patients with mitral valve disease ${ }^{12}$ the incidence of left atrial thrombus ranged from $9 \%$ to $17 \%$, and episodes of systemic embolisation were reported in $29 \%$ to $60 \%$ of patients with such thrombosis. ${ }^{2}$ In mitral valve disease, therefore, the detection of a left atrial thrombus and evaluation of its haematological activity are essential for determining the need for anticoagulant treatment and the timing of open heart surgery. Detection of left atrial thrombi has been attempted using angiocardiography, ${ }^{2}$ cross sectional echocardiography, ${ }^{3}$ and computed tomography. ${ }^{4}$ Angiocardiography and echocardiography have limitations, however, depending on the size and location of the thrombus. On the other hand, computed tomography can accurately detect the presence of left atrial thrombi. ${ }^{4}$ These methods cannot, however, determine whether the thrombi are haematologically active; this needs to be known to determine the

Requests for reprints to Dr Makoto Yamada, Heart Centre, Osaka Prefectural Hospital, 1-56 3-chome, Mandai-higashi, Sumiyoshi-ku, Osaka, 558 Japan.

Accepted for publication 1 November 1983 efficacy of anticoagulant treatment.

Recently, in vitro labelling of autologous platelets with indium-111 oxine was performed by Thakur $e t$ al. ${ }^{5}$ Their method looks promising for assessing the haematological activity of thrombi in vivo. Some studies in animals have shown that the indium-111 labelling method is useful in the diagnosis of infective endocarditis ${ }^{6}$ and coronary thrombosis. ${ }^{7}$ Deep vein thrombosis ${ }^{8}$ and systemic artery thrombi ${ }^{9}$ have also been detected in man by this technique. Furthermore, by comparison of indium-111 oxine platelet imaging with findings at operation the technique has been shown to be valuable in detecting active thrombi in left ventricular aneurysms complicating myocardial infarction. ${ }^{1011}$ Nevertheless, few attempts have been made to detect left atrial thrombi by this technique because (a) the time of the formation of the left atrial $\sigma$ thrombus is usually unclear and (b) it is also uncertain 0

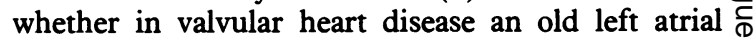
thrombus is haematologically active. Moreover, in $\stackrel{?}{?}$ order to detect by this technique a left atrial $\underset{\square}{ }$ thrombus-particularly one confined to the left atrial $\stackrel{\circ}{\circ}$ appendage-radionuclide images of high spatial $\stackrel{\Omega}{\stackrel{D}{\perp}}$ resolution are necessary. In the present study we $\stackrel{\mathbb{D}}{\mathbb{D}}$ attempted to detect left atrial thrombi using indium- $\frac{\sigma}{\sigma}$ 
Table 1 Clinical data and results of indium-III platelet scintigraphy in 28 patients with mitral and combined valve diseases

\begin{tabular}{|c|c|c|c|c|c|c|c|}
\hline $\begin{array}{l}\text { Case } \\
\text { No }\end{array}$ & $\begin{array}{l}\text { Age } \\
\text { (yr) }\end{array}$ & Sex & Diagnosis* & $\begin{array}{l}\text { Clinical } \\
\text { emboli }\end{array}$ & $\begin{array}{l}\text { Platelet } \\
\text { scan }\end{array}$ & $\begin{array}{l}\text { Clot (wt) at } \\
\text { operation or } \\
\text { necropsy }\end{array}$ & $\begin{array}{l}\text { Labelling } \\
\text { efficiency } \\
\text { (\%) }\end{array}$ \\
\hline $\begin{array}{l}1 \\
2 \\
3 \\
4 \\
5 \\
6 \\
7 \\
8 \\
9 \\
10 \\
11 \\
12 \\
13 \\
14^{\star} \\
15 \\
16 \\
17 \\
18 \\
19 \\
20 \\
21 \\
22 \\
23 \\
24 \\
25 \\
26 \\
27 \\
28\end{array}$ & $\begin{array}{l}46 \\
63 \\
54 \\
45 \\
65 \\
46 \\
69 \\
55 \\
26 \\
60 \\
63 \\
43 \\
58 \\
66 \\
55 \\
52 \\
50 \\
47 \\
67 \\
54 \\
56 \\
66 \\
51 \\
75 \\
61 \\
51 \\
45 \\
52\end{array}$ & $\begin{array}{l}\mathbf{F} \\
\mathbf{F} \\
\mathbf{F} \\
\mathbf{F} \\
\mathbf{M} \\
\mathbf{M} \\
\mathbf{M} \\
\mathbf{F} \\
\mathbf{F} \\
\mathbf{F} \\
\mathbf{F} \\
\mathbf{M} \\
\mathbf{F} \\
\mathbf{F} \\
\mathbf{F} \\
\mathbf{F} \\
\mathbf{F} \\
\mathbf{M} \\
\mathbf{M} \\
\mathbf{F} \\
\mathbf{F} \\
\mathbf{F} \\
\mathbf{M} \\
\mathbf{F} \\
\mathbf{F} \\
\mathbf{F} \\
\mathbf{M} \\
\mathbf{F}\end{array}$ & $\begin{array}{l}\text { MS } \\
\text { MS } \\
\text { MS } \\
\text { MSR } \\
\text { MS } \\
\text { MS } \\
\text { MS } \\
\text { MSR } \\
\text { MS } \\
\text { MSR } \\
\text { MSR } \\
\text { MS } \\
\text { MS } \\
\text { MS } \\
\text { MS, AR } \\
\text { MSR, ASR } \\
\text { MSR, ASR } \\
\text { MS, AR } \\
\text { MS, AR } \\
\text { MSR, ASR } \\
\text { MSR, ASR } \\
\text { MSR, ASR } \\
\text { MSR, ASR } \\
\text { MSR, ASR } \\
\text { MS,ASR } \\
\text { MSR, ASR } \\
\text { MS,AR } \\
\text { MSR, ASR }\end{array}$ & $\begin{array}{l}\text { None } \\
\text { None } \\
\text { None } \\
\text { None } \\
\text { Cerebral } \\
\text { None } \\
\text { None } \\
\text { None } \\
\text { None } \\
\text { None } \\
\text { None } \\
\text { None } \\
\text { None } \\
\text { None } \\
\text { None } \\
\text { None } \\
\text { None } \\
\text { Limbs } \\
\text { Cerebral } \\
\text { None } \\
\text { Cerebral } \\
\text { Cerebral } \\
\text { None } \\
\text { None } \\
\text { None } \\
\text { None } \\
\text { Cerebral } \\
\text { None }\end{array}$ & $\begin{array}{l}- \\
+ \\
- \\
- \\
- \\
- \\
+ \\
- \\
- \\
- \\
- \\
- \\
- \\
- \\
- \\
- \\
- \\
- \\
+ \\
- \\
+ \\
+ \\
- \\
+ \\
+ \\
+ \\
-\end{array}$ & $\begin{array}{l}\text { ND } \\
+(66 \text { g) } \\
\text { ND } \\
\text { ND } \\
\text { ND } \\
\text { ND } \\
\text { ND } \\
\text { ND } \\
\text { ND } \\
\text { ND } \\
\text { + } \\
\text { ND } \\
\text { ND } \\
\text { ND } \\
\text { - } \\
\text { ND } \\
\text { +(44 g) } \\
\text { ND } \\
\text { ND } \\
\text { ND } \\
\text { +(40 g) } \\
\text { + } \\
\text { + }\end{array}$ & $\begin{array}{l}53 \\
41 \\
46 \\
56 \\
54 \\
63 \\
53 \\
49 \\
67 \\
38 \\
34 \\
60 \\
16 \\
49 \\
61 \\
56 \\
38 \\
25 \\
51 \\
22 \\
34 \\
49 \\
51 \\
47 \\
33 \\
26 \\
21 \\
55\end{array}$ \\
\hline
\end{tabular}

MS, mitral stenosis; MSR, mitral stenosis and regurgitation; AR, aortic regurgitation; ASR, aortic stenosis and regurgitation; ND, not done; + , positive findings; - , negative findings.

$\star$ All patients except case 14 also had atrial fibrillation.

111 oxine platelet imaging and compared the results obtained with the surgical and necropsy findings.

\section{Patients and methods}

Twenty eight patients with valvular heart disease were studied (eight men and 20 women; mean $( \pm S D)$ age $55 \cdot 0 \pm 10 \cdot 1$ years (range $26-75$ years)) (Table 1 ). All the patients gave informed consent. Of the 28 patients, 14 had mitral valve disease and 14 combined valve disease. Twenty seven of them had atrial fibrillation. Mitral valve disease and combined valve disease were diagnosed by angiocardiography or cross sectional echocardiography or both. Four of the 14 patients with mitral valve disease and seven of the 14 with combined valve disease underwent open heart surgery. One patient with combined valve disease died of heart failure before operation; a necropsy was performed. The interval between the last radionuclide image and heart surgery or necropsy ranged from one to 60 (mean $20.5 \pm 19.9$ ) days.

\section{PREPARATION OF INDIUM-111 LABELLED}

PLATELETS

Platelet labelling was performed using commercially available indium-111 oxine (Amersham International
Limited, London) or indium-111 oxine prepared in our laboratory by the method of Scheffel et al. ${ }^{12}$ In vivo labelling was carried out by the slightly modified button saline method of Wistow et al. ${ }^{13}$ Acid citrate dextrose (ACD) and ACD saline (pH 6.5) solution were sterilised by millipore filtration. Forty three millilitres of whole blood were collected from the antecubital vein through an 18 gauge needle into $7 \mathrm{ml}$ of the ACD solution. After being mixed with the solution the blood was transferred to six sterile plastic disposable tubes and centrifuged at $200 \mathrm{~g}$ for 10 minutes. The upper three quarters of the platelet rich plasma of each tube was transferred with sterile plastic pipettes into two plastic tubes, and one twentieth of its volume of ACD solution was added to each tube. This platelet rich plasma was then centrifuged at 1000 $g$ for 10 minutes. After the resulting platelet poor plasma had been saved, the cell button (platelets) was cleansed with ACD saline solution, resuspended in $1.5-2.0 \mathrm{ml}$ of ACD saline solution containing indium-111 oxine complex $(916 \pm 52 \mu \mathrm{Ci}(33.9 \pm 1.9$ $\mathrm{MBq})$ ) and incubated at $37^{\circ} \mathrm{C}$ for three to five minutes. The labelled platelet suspension was mixed with an equal volume of platelet poor plasma and centrifuged at $1000 \mathrm{~g}$ for 10 minutes. The supernatant was carefully decanted and the labelled platelet button finally resuspended in $8 \mathrm{ml}$ of platelet poor plasma. 
The indium-111 activity remaining in the platelets was determined to assess the efficiency of the in vitro labelling. The platelet suspension was injected intravenously using a 21 gauge needle.

\section{RADIONUCLIDE IMAGING}

Usually, images were obtained between two and five and at 48 and 96 hours after injection of the labelled platelets. In case 20 (Table 1) images were obtained 72 hours after injection instead of at 96 hours. All patients were examined in the anterior, right anterior oblique $\left(45^{\circ}\right)$, and left lateral views. Imaging was performed on a large field of view gamma scintillation camera (Technicare $\Sigma 410$ S) fitted with a middle energy collimator and set on both photopeaks (173 $\mathrm{keV}$ and $247 \mathrm{keV}$ ) of indium-111 with a $20 \%$ window. In all patients, 300000 count images were obtained.

\section{CRITERION FOR INTERPRETATION OF PLATELET} IMAGES

All images were interpreted by two observers without knowledge of the clinical and laboratory data by the following criterion. When scintiphotos obtained in two or three views 72 or 96 hours after injection of the labelled platelets showed "hot spot areas"-defined as areas of increased and abnormal indium-111 activity within the left atrial blood pool-and the activity in these areas did not decrease with time the images were interpreted as positive for thrombi within the left atrium.

\section{Results}

In all patients well defined images of the cardiac blood pool and great vessels were seen two to five hours after injection of labelled platelets. The results are shown in Table 1 . Of the 28 patients, seven had positive platelet images and 21 negative images. In case 2 large "hot spot areas" were seen 48 and 96 hours after injection at a position corresponding to the left atrium (Fig. la), and the images were interpreted as positive. Mitral valve replacement was performed, and thrombi

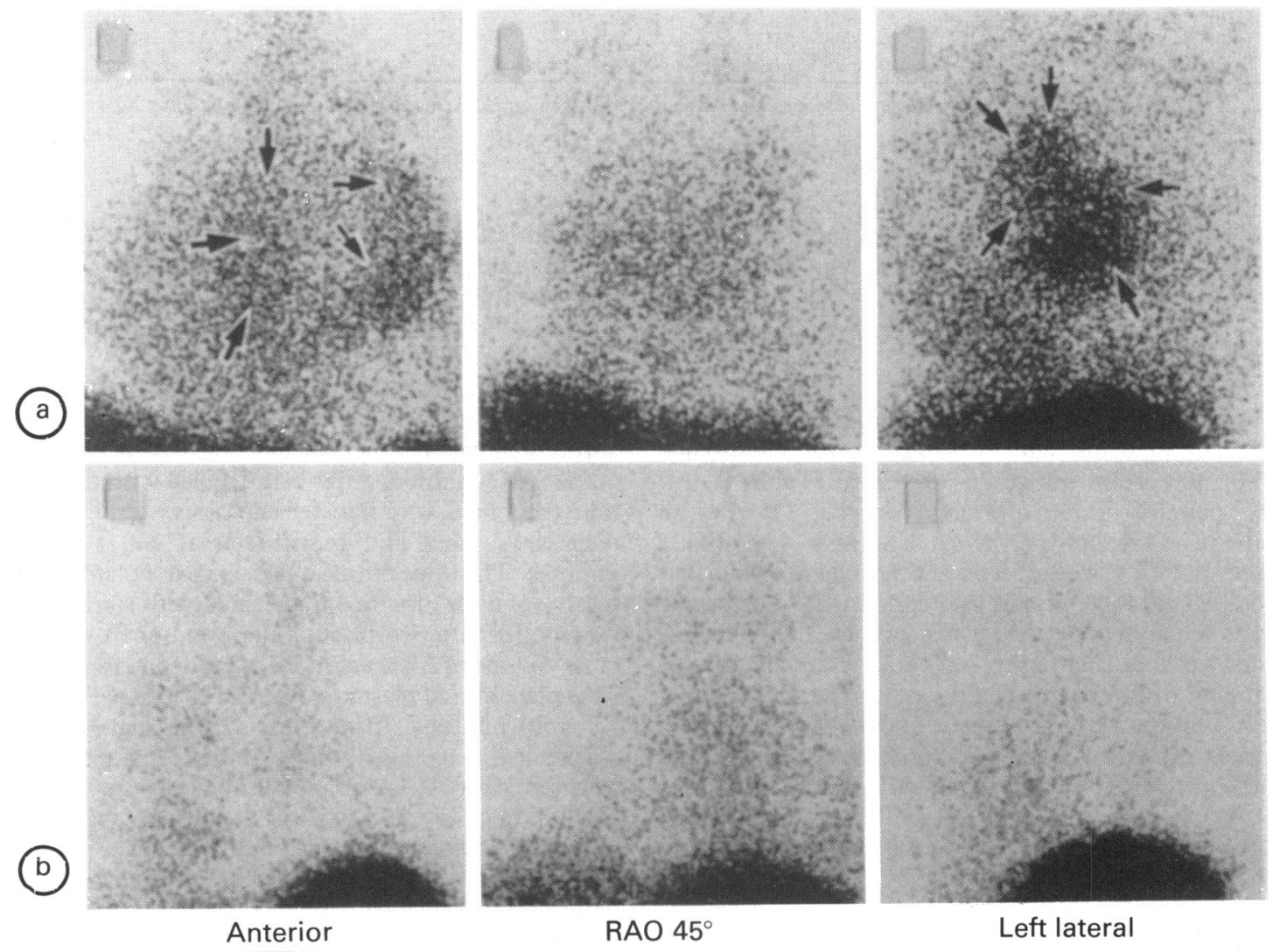

Fig. 1 Indium-111 scintiphotos obtained 96 hours after injection of labelled platelets (case 2). (a) In the anterior and left lateral views "hot spot areas" of increased and abnormal activity (arrows) represent active thrombi within the left atrium. (b) Scintiphotos obtained three months after surgery showed that no "hot spot area" was present. RAO, right anterior oblique. 

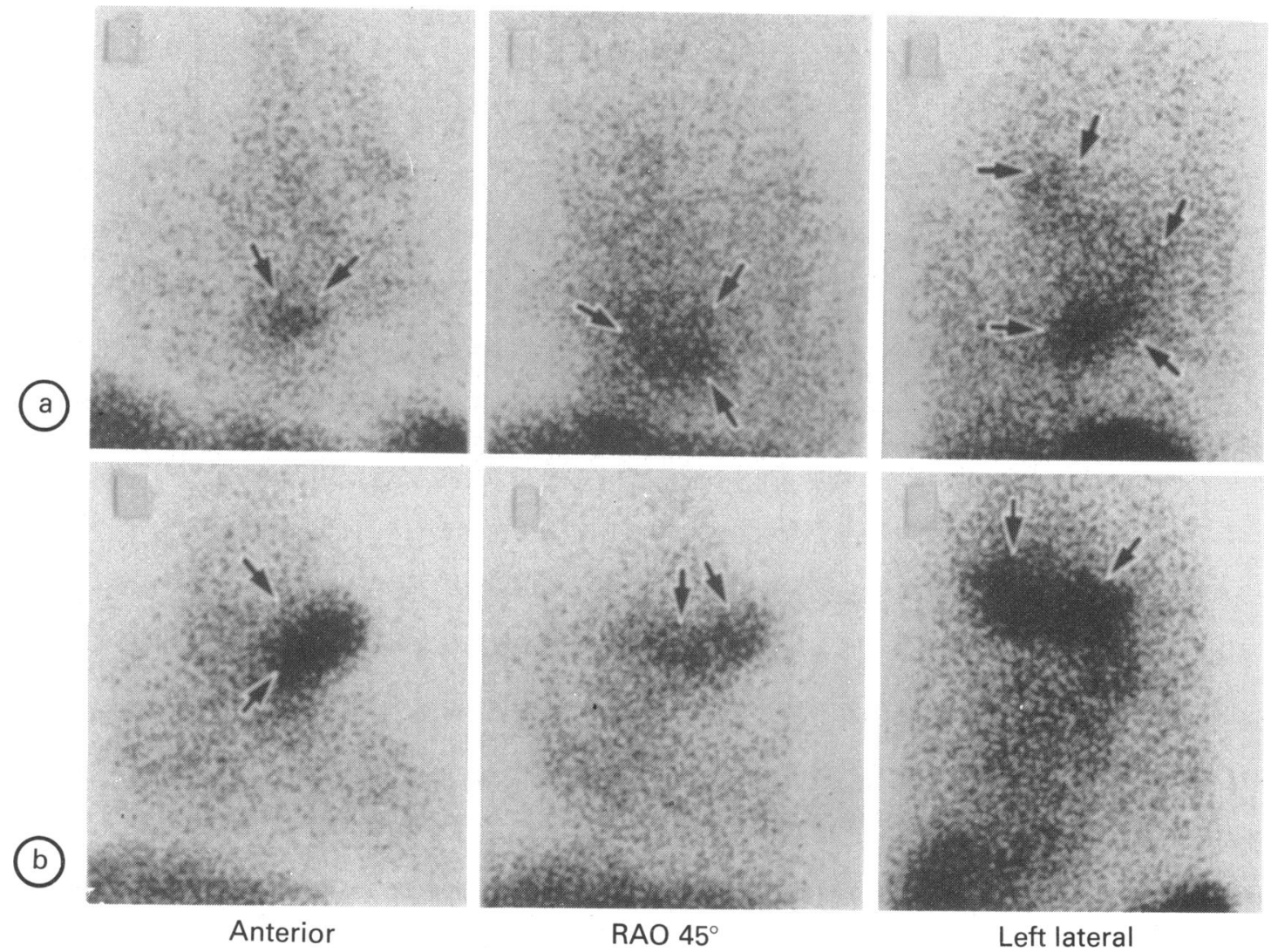

Fig. 2 Indium-111 scintiphotos obtained (a) 72 hours after injection of labelled platelets (case 20) showing "hot spot areas" (arrows) within the cardiac blood pool corresponding to the left atrium in anterior, $R A O 45^{\circ}$, and left lateral views; and (b) 96 hours after injection of labelled platelets (case 25) showing "hot spot areas" (arrows) in anterior, RAO 45', and left lateral views.

weighing about $66 \mathrm{~g}$ were identified in the area stretching from the left atrial posterior wall to the left atrial appendage. To determine whether the "hot spot areas" in case 2 corresponded to intra-atrial thrombi indium-111 labelled platelets were injected intravenously three months after operation and negative platelet images were obtained (Fig. 1b). In case 20 positive platelet images were obtained (Fig. 2a). Mitral valve replacement, aortic valve repair, and left atrial plication were performed and at operation thrombi of about $44 \mathrm{~g}$ were seen in the area from the left atrial posterior wall to the left atrial appendage. In case 25 positive platelet images were obtained (Fig. $2 \mathrm{~b}$ ). The patient died of heart failure before operation could be performed, and at necropsy thrombi weighing about $40 \mathrm{~g}$ were identified in the same area. In case 27 "hot spot areas" corresponding to the left atrial appendage were seen 48 and 96 hours after injection of the labelled platelets (Fig. 3), and the images therefore were interpreted as positive. The patient under- went open mitral commissurotomy and left atrial appendage closure. At operation a thrombus was found in the left atrial appendage. In cases 7, 22, and 26 , in which the scintiphotos were interpreted as positive, the presence or absence of thrombi was not directly confirmed because no surgical procedures were performed. In case 22 , however, the patient had a history of cerebral infarction suggesting that there might be a thrombus within the left atrium.

Although "hot spot areas" were observed 48 and 96 hours after the platelet injection in case 18 (Fig. 4) the images were interpreted as negative for a thrombus within the left atrium since the areas were seen only in the left lateral view and indium-111 activity occurring 96 hours after injection showed a slight decrease compared with that after 48 hours. No thrombus was identified at operation. In case 21 only a scintiphoto taken in the left lateral view 96 hours after injection showed a "hot spot area." The image was, therefore, interpreted as negative; no thrombus was identified 
Fig. 3 Indium-111 scintiphotos obtained (a) 48 and (b) 96 hours after injection of labelled platelets (case 27) showing "hot spot areas" in anterior and left lateral views. Note that indium-111 activity in the "hot spot areas" increased with time.

at operation. In cases $9,13,17,23$, and 28 "hot spot areas" were not observed in any of the three views, and the images were interpreted as negative; no thrombus was identified at operation. In case 12 scintiphotos were interpreted as negative since no "hot spot areas" were observed 96 hours after the platelet injection. This patient underwent open mitral commissurotomy and left atrial appendage closure as a thombus was identified at operation.

\section{Discussion}

In the present method, the platelet preparation consisted of $2.44 \pm 0.55 \times 10^{9}$ (mean \pm SD) platelets with an indium-111 activity of $405 \pm 132 \mu \mathrm{Ci}(15 \pm 4.9$ $\mathrm{MBq})(\mathrm{mean} \pm \mathrm{SD})$ and a final labelling efficiency of $44.6 \pm 13.6 \%$ (mean $\pm S D)$. Well defined images of the cardiac blood pool were obtained in all cases.

As far as we know, the present study is the first to detect left atrial thrombi using indium-111 platelet imaging in man. Of seven cases that were interpreted as having positive platelet images thrombi were confirmed at operation or necropsy in four. Of 21 cases that were interpreted as having negative platelet images, no thrombus was confirmed at operation in seven and one had a false negative result.

In the four cases with positive platelet images the surface of thrombi appeared red and friable when examined at operation or necropsy. These findings indicate that the left atrial thrombi in these patients with long term valvular disease were haematologically active and were detected by indium-111 platelet scintigraphy.

In the present study two cases (cases 18 and 21) might have been misinterpreted as positive since "hot spot areas" were seen in the left lateral view. In case 18 "hot spot areas" were seen 48 and 96 hours after injection of labelled platelets only in the left lateral view (Fig. 4). Nevertheless, indium-111 activity in the "hot spot area" decreased during the period from 48 to 96 hours after the injection (Fig. 4). In contrast, in all the cases in which scintiphotos were interpreted as positive and thrombi within the left atrium were identified at operation indium-111 activity in the "hot spot areas" increased with time after injection of the labelled platelets. Note the increased indium-111 activity on the scintiphotos in case 27 (Fig. 3). These results indicate that the labelled platelets accumulated 
(a)

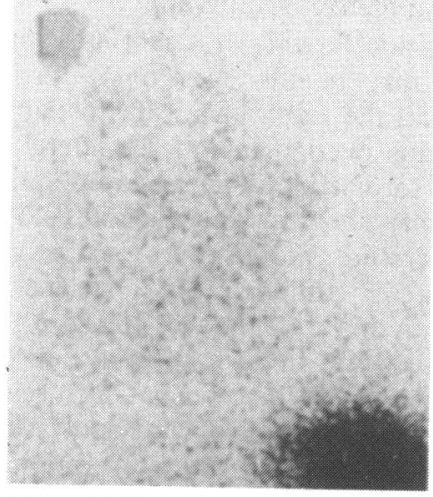

(b)

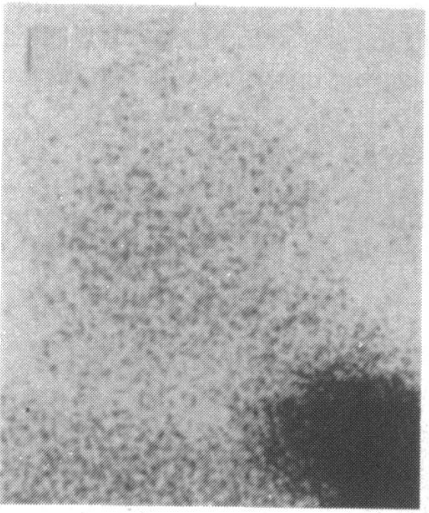

Anterior
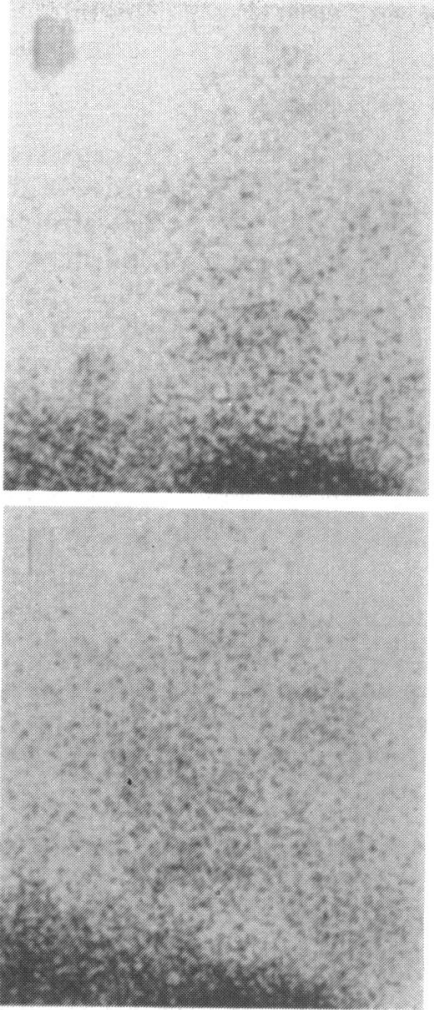

RAO $45^{\circ}$
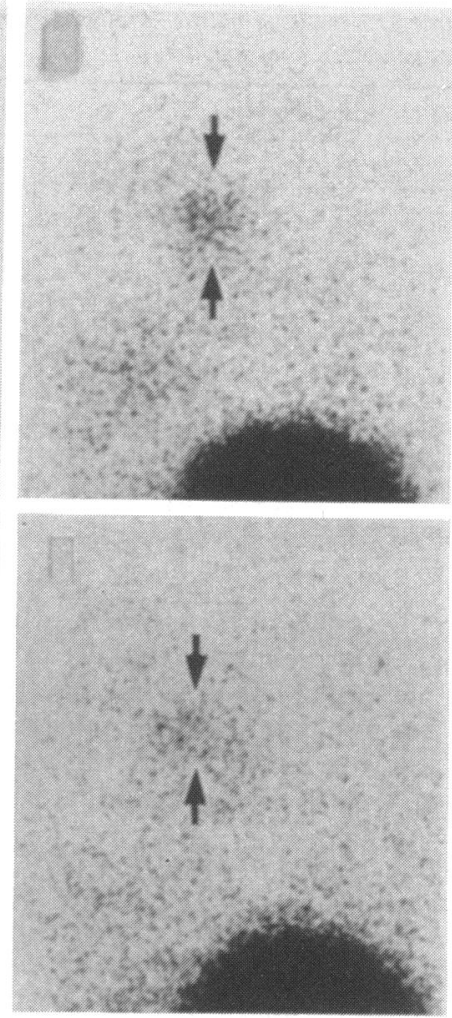

Left lateral

Fig. 4 Indium-111 scintiphotos obtained (a) 48 and (b) 96 hours after injection of labelled platelets (case 18) showing "hot spot areas" (arrows) only in the left lateral views. Note that indium-111 activity in the "hot spot area" decreased with time during the period from 48 to 96 hours.
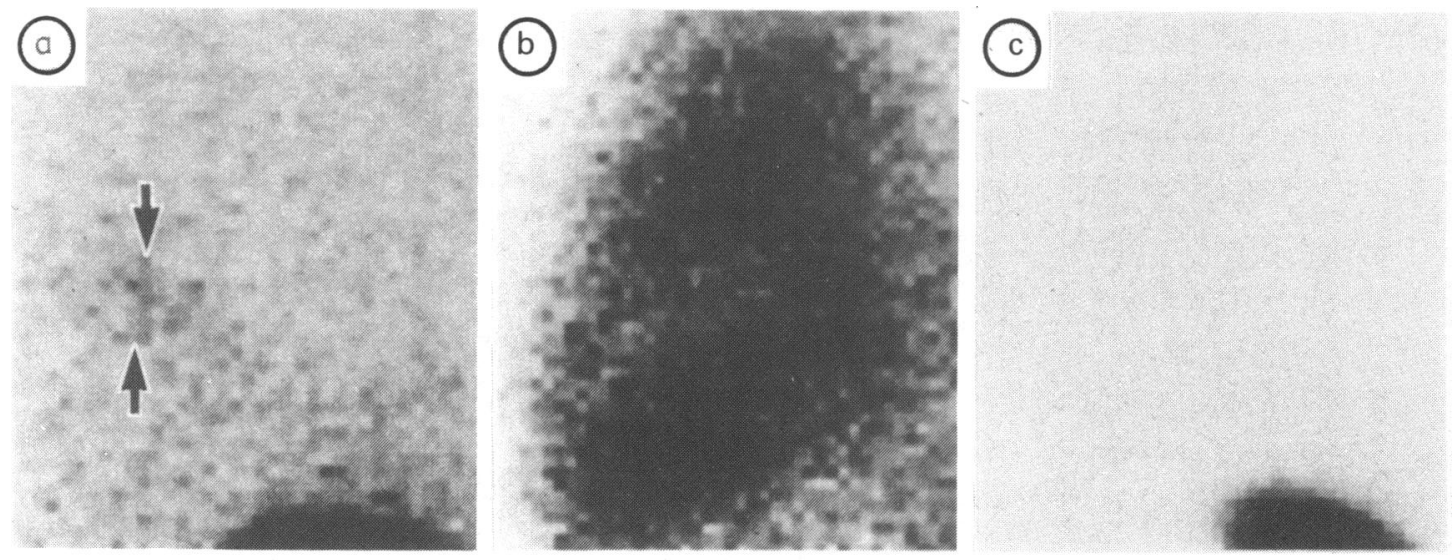

Left lateral view

Fig. 5 (a) Indium-111 scintiphoto obtained 96 hours after injection of labelled platelets and (b) technetium-99m blood pool image. Note that the "hot spot area" (arrows) in (a) disappeared in the subtraction image (c) of the blood pool (subtraction of technetium blood pool image from indium-111 scintiphoto). 
Table 2 Results of platelet scintigraphy in nine patients whose images showed "hot spot areas"

\begin{tabular}{lllll}
\hline $\begin{array}{l}\text { Case } \\
\text { No }\end{array}$ & \multicolumn{2}{l}{ Imaging view } & & $\begin{array}{l}\text { Initial imaging } \\
\text { time }(\boldsymbol{h})^{\star}\end{array}$ \\
\cline { 2 - 5 } & $\boldsymbol{A}$ & $\boldsymbol{R} A O$ & $L L$ & \\
\hline 2 & + & - & + & 48 \\
7 & + & + & - & 48 \\
18 & - & - & + & 48 \\
20 & + & + & + & 48 \\
21 & - & - & + & 96 \\
22 & + & - & + & 48 \\
25 & + & + & + & 48 \\
26 & + & - & + & 48 \\
27 & + & - & + & 48 \\
\hline
\end{tabular}

A, anterior; RAO, right anterior oblique $\left(45^{\circ}\right)$; LL, left lateral; +, positive findings; - , negative findings.

$\star$ Initial imaging time was taken as the time when "hot spot areas" were first seen.

on the surface of the thrombus with time. It is, therefore, very important clinically to assess the presence or absence of left atrial thrombi from images taken at different points in time. Similar results were found by Ezekowitz et al who detected intraventricular thrombi using platelet scintigraphy. ${ }^{14}$

An explanation for the "hot spot area" in the left lateral view may be the overlap of the left atrial appendage and the right atrium in this lateral view. Because of this overlap in the left lateral view the image of the cardiac blood pool corresponding to the left atrium may show increased indium-111 activity and may, therefore, be misinterpreted as showing a "hot spot area," although the platelets have not accumulated in this area. In cases 18 and 21 "hot spot areas" were seen only in the left lateral view but not in the others (Fig. 4). In the cases in which scintiphotos were interpreted as positive "hot spot areas" were seen in two or three views (Table 2). In four of these cases thrombi were identified at operation or necropsy. In fact, in case 18 indium-111 labelled platelets were injected intravenously two months after operation, and a "hot spot area" was seen 96 hours after injection only in the left lateral view. Immediately after scintiphotos had been obtained 96 hours after the platelet injection technetium-99m labelled human serum albumin was injected intravenously. As shown

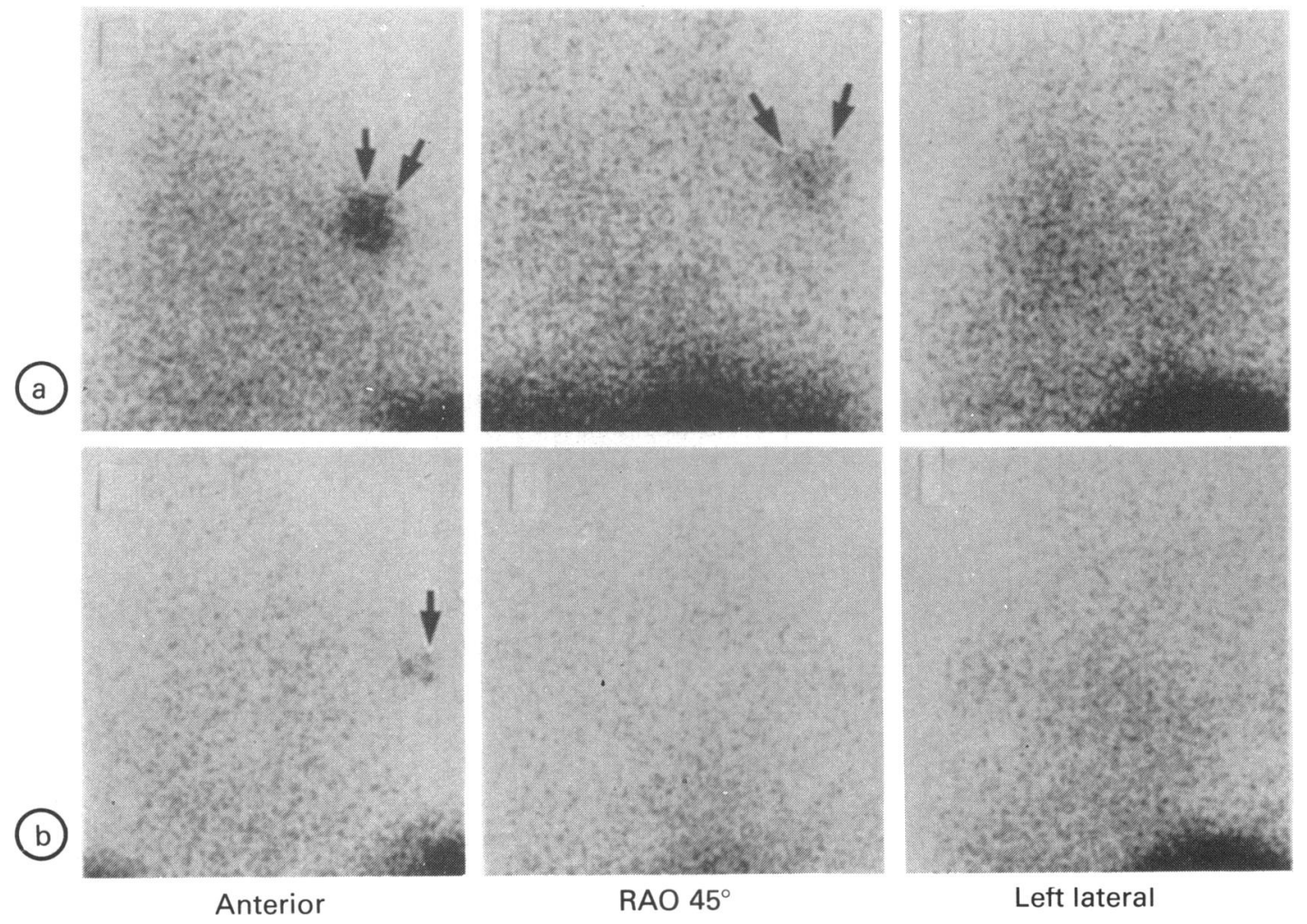

Fig. 6 Indium-111 scintiphotos obtained (a) before and (b) after warfarin treatment showing that the "hot spot areas" (arrows in (a)) notably decreased after warfarin treatment. Note that the "hot spot area" seen in the RAO $45^{\circ}$ view in (a) disappeared completely in $(b)$. 
in Fig. 5, the "hot spot area" seen in the left lateral view disappeared after subtraction of the blood pool according to Bergmann $e t a l$ and Sutherland et al 1516 (slightly modified). This result indicates that the "hot spot area" seen in the left lateral view in case 18 could not have reflected platelet deposition on to a thrombus. Great caution should, however, be taken in interpreting images in only one view, especially the left lateral view.

Case 12 showed a false negative result. In this case, high indium-111 activity in the cardiac blood pool did not decrease substantially 96 hours after injection of the labelled platelets, and such high activity may lead to misinterpretation. Images should, therefore, have been obtained later than 96 hours after the platelet injection in this case.

These results show that two important factors may affect the accuracy of indium-111 platelet scintigraphy in detecting left atrial thrombi: (a) the high indium-111 activity in the cardiac blood pool (background) and (b) the artificially high activity in the left atrial blood pool caused by the overlap of the left atrial appendage and the right atrium in the left lateral view. Interpretation, using time sequential images, is, therefore, essential to detect left atrial thrombi accurately. Using our criterion of positivity, therefore, the accuracy in detecting left atrial thrombi by indium111 platelet scintigraphy was $92 \%$ as confirmed by surgical and postmortem examinations.

In addition, the reliability of this criterion for detecting left atrial thrombi was tested by the following procedure. To determine whether "hot spot areas" actually reflect the localisation of labelled platelets on to the surface of the thrombus an anticoagulant agent ${ }^{1718}$ was used. A patient with combined valve disease whose scintiphotos were interpreted as positive (Fig. 6a) was treated with warfarin for about one month to reduce the prothrombin time to $37 \%$. Indium-111 labelled platelets were injected intravenously after treatment with the warfarin, and indium-111 activity in the "hot spot areas" was considerably decreased (Fig. 6b) suggesting that the "hot spot areas" do reflect the accumulation of the labelled platelets on to the thrombus. These results strongly suggest that the present criterion is reliable for detecting haematological activity in a left atrial thrombus and that indium-111 platelet scintigraphy is an excellent method of detecting haematologically active thrombi within the left atrium associated with long term valvular disease.

We thank Drs Yoshinari Isaka and Hideki Etani for their valuable comments and suggestions during the course of this study. We also thank Dr Hiroshi Kita for his help during preparation of the manuscript and Miss Aki Katayama for her technical assistance.

\section{References}

1 Parker BM, Friedenberg MJ, Templeton AW, Burford TH. Preperative angiocardiographic diagnosis of left atrial thrombi in mitral stenosis. $N$ Engl f Med 1965; 273: 136-40.

2 Lewis KB, Criley JM, Ross RS. Detection of left atrial thrombus by cineangiocardiography. Am Heart $\mathcal{f}$ 1965; 70: 612-9.

3 Mikell FL, Asinger RW, Rourke T, Hodges M, Sharma B, Francis GS. Two-dimensional echocardiographic demonstration of left atrial thrombi in patients with prosthetic mitral valves. Circulation 1979; 60: 1183-90.

4 Tomoda H, Hoshiai M, Furuya H, et al. Evaluation of intracardiac thrombus with computed tomography. Am $\mathcal{F}$ Cardiol 1983; 51: 843-52.

5 Thakur ML, Welch MJ, Joist JH, Coleman RE. Indium-111labelled platelets: studies on preparation and evaluation of in vitro and in vivo functions. Thromb Res 1976; 9: 345-57.

6 Riba AL, Thakur ML, Gottschalk A, Andriole VT, Zaret BL. Imaging experimental infective endocarditis with indium-111labelled blood cellular components. Circulation 1979; 59: 336-43.

7 Riba AL, Thakur ML, Gottschalk A, Zaret BL. Imaging experimental coronary artery thrombosis with indium-111 platelets. Circulation 1979; 60: 767-75.

8 Goodwin DA, Bushberg JT, Doherty PW, et al. Indium-111labelled autologous platelets for location of vascular thrombi in humans. F Nucl Med 1978; 19: 626-34.

9 Davis HH, Heaton WA, Siegel BA, et al. Scintigraphic detection of atherosclerotic lesions and venous thrombi in man by indium111-labelled autologous platelets. Lancet 1978; i: 1185-7.

10 Ezekowitz MD, Leonard JC, Smith EO, Allen EW, Taylor FB. Identification of left ventricular thrombi in man using indium111-labelled autologous platelets. Circulation 1981; 63: 803-10.

11 Stratton JR, Ritchie JL, Hamilton GW, Hammermeister KE, Harker LA. Left ventricular thrombi: in vitro detection by indium-111 platelet imaging and two-dimensional echocardiography. Am $\mathcal{f}$ Cardiol 1981; 47: 874-81.

12 Scheffel U, McIntyre PA, Evatt B, et al. Evaluation of indium111 as a new high photon yield gamma-emitting 'physiological' platelet label. Fohns Hopkins Med f 1977; 140: 285-93.

13 Wistow BW, Grossman ZD, McAfee JG, Subramanian G, Henderson RW, Roskopf ML. Labelling of platelets with oxine complexes of Tc-99m and In-111. Part 1. In vitro studies and survival in the rabbit. $\mathcal{F}$ Nucl Med 1978; 19: 483-7.

14 Ezekowitz MD, Burrow RD, Heath PW, Streitz T, Smith EO, Parker DE. Diagnostic accuracy of indium-111 platelet scintigraphy in identifying left ventricular thrombi. Am $\mathcal{F}$ Cardiol 1983; 51: $1712-6$.

15 Bergmann SR, Lerch RA, Mathias CJ, Sobel BE, Welch MJ. Noninvasive detection of coronary thrombi with In-111 platelets: concise communication. 7 Nucl Med 1983; 24: 130-5.

16 Sutherland GR, King ME, Peerless SJ, Vezina WC, Brown GW, Chamberlain MJ. Platelet interaction within giant intracranial aneurysms. F Neurosurg 1982; 56: 53-61.

17 Fedullo PF, Moser KM, Moser KS, Konopka R, Hartman MT. Indium-111-labelled platelets: effect of heparin on uptake by venous thrombi and relationship to the activated partial thromboplasin time. Circulation 1982; 66: 632-7.

18 Pumphrey CW, Chesebro JH, Dewanjee MK, et al. In vivo quantitation of platelet deposition on human peripheral arterial bypass grafts using indium-111-labelled platelets. Am $\mathcal{F}$ Cardiol 1983; 51: 796-801. 